\section{The current version of the visceral adiposity index is not suitable for application in pediatric populations: comments on the article by Al-Daghri et al.}

\author{
doi: 10.1038/pr.2014.98
}

To the Editor: Al-Daghri et al. (1) have investigated the suitability of the visceral adiposity index (VAI) as a marker of early cardiometabolic risk in children and adolescents. The authors conclude that the VAI shows a less marked association than BMI with insulin sensitivity, adipokines, and subclinical inflammation. Therefore, they recommend caution in applying the VAI in children.

We consider that the aim of the authors is very appropriate, and the conclusions are also correct. However, there is only one big underlying problem: the current version of the VAI is not suitable for application in pediatric populations.

The VAI is a gender-specific mathematical index, based on simple anthropometric (BMI and waist circumference (WC)) and metabolic parameters (triglycerides and high-density lipoprotein cholesterol). It is an empirical model that does not originate from theoretical assumptions but from the observation in a healthy normal/overweight adult population of a linear relationship between BMI and WC, from which a linear equation has been extrapolated (2,3). A model of adipose distribution was initially created based on this linear equation, and then, it was corrected for triglycerides and high-density lipoprotein cholesterol, determining the VAI.

Therefore, the numerical constants that we find in the formula refer to the relationship between BMI and WC and to the mean triglycerides and high-density lipoprotein cholesterol levels, in a healthy adult Caucasian population. It is not correct to apply the current version of the VAI in non-Caucasian and pediatric patients.

Future studies should study the WC/BMI relationship and the mean levels of triglycerides and high-density lipoprotein cholesterol in large healthy pediatric populations, in order to formulate (using the same procedure for our modeling) a "pediatric VAI."

We believe that investigating the cardiometabolic risk in children is of great importance, since at this stage, it is not always detectable as an overt metabolic syndrome.

In this regard, we inform you that our research team is collecting a large series of healthy children and adolescents to formulate a pediatric VAI.

In future, the use of a reliable index can distinguish "metabolically healthy obesity" from "at metabolic risk obesity," and it will be of fundamental importance to settle the still open question of pediatric obesity as an independent predictor of cardiometabolic risk in adulthood.

Disclosure: The authors have nothing to disclose.

\section{Marco C. Amato' and Carla Giordano ${ }^{1}$}

${ }^{1}$ Dipartimento Biomedico di Medicina Interna e Specialistica (Dibimis), Section of Cardio-Respiratory and Endocrine-Metabolic Diseases, University of Palermo, Palermo, Italy.

Correspondence: Carla Giordano (carla.giordano@unipa.it)

\section{REFERENCES}

1. Al-Daghri NM, Al-Attas OS, Alokail M, et al. Does visceral adiposity index signify early metabolic risk in children and adolescents?: Association with insulin resistance, adipokines, and subclinical inflammation. Pediatr Res 2014;75:459-63.

2. Amato MC, Giordano C, Galia M, et al. Visceral Adiposity Index: a reliable indicator of visceral fat function associated with cardiometabolic risk. Diabetes Care 2010;33:920-2.

3. Amato MC, Giordano C. Clinical indications and proper use of Visceral Adiposity Index. Nutr Metab Cardiovasc Dis 2013;23:e31-2. 